\title{
Fundamentals of Implant Overdenture-Overview
}

\author{
Hariharan Ramakrishnan ${ }^{1 *}$ and Maniamuthu Ragupathi ${ }^{2}$ \\ ${ }^{1}$ Professor, Department of Prosthodontics \& Implantology, Ragas Dental College \&Hospital, MGR Medical University, Chennai, India \\ ${ }^{2}$ Lecturer, Ragas dental college and hospital, Chennai, India
}

ORCID: 0000-0003-4466-5744

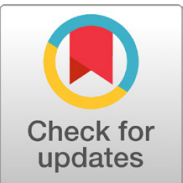

*Corresponding author: Hariharan Ramakrishnan, Professor, Department of Prosthodontics and Implantology, Ragas Dental college and Hospital, MGR Medical University, Chennai, Tamilnadu, India.Email: abcv2005@yahoo.com.

Received Date: February 19, 2021

Published Date: March 24, 2021

\begin{abstract}
Edentulism is a condition where there is loss of teeth, that changes the lifestyle of a human and results in various systemic changes. Preservation of remaining structures are important and implant overdentures are a boon not only in replacing the lost oral structures but also restores esthetics and functions. This aids in enhancing the nutritional intake and normalise the quality of life of the patient. After the loss of natural teeth restoring the oral structures with the support of implants is both an art and science, which will be described in this article.
\end{abstract}

Keywords: Denture; Overdenture; Dental implant; Bone density; Dental prosthesis; Mandible; Maxilla

\section{Introduction}

The state of tooth loss is a common problem due to various causes such as periodontitis, systemic diseases, oral pathology, dental caries. Awareness about oral hygiene and care helps improve the dentate status of the patient. Preservation of remaining oral structures is also important in prosthodontic rehabilitation. Overdentures play a vital role in preservation of remaining tooth, tooth root and alveolar bone. The main benefits of overdenture are retention and stability of prosthesis, which gives the psychologic benefit.

Introduction of implants and their proven results of osseointegration by Branemark et al., at Toronto conference in 1982 had changed the entire trend of dentistry. For a completely edentulous, implants may be used as a retentive tool for overdentures that gives all the advantages of tooth supported overdentures. Implant supported restorations are of two types. Implant supported fixed restoration and implant retained overdenture. Implant supported restorations are mostly advised in low to moderate bone atrophy cases while implant retained overdentures are advised in case of high bone atrophy [1,2]. Implant retained overdentures are cost effective and maintainence is also easier compared to implant supported restorations. Long term success rate and easy convertibility of implant overdenture to conventional complete dentures in case of implant failures are the added benefits [2,3]. In case of maxilla 4 to 6 implants are needed for retention, in mandible two implants supported over denture or single implant retained over denture is also advisable $[2,4]$. The minimum number of implants needed for an over denture is still in debate. In this article, the aid to achieve good prognosis such as patient selection, diagnosis, treatment planning are discussed.

\section{Description of history of overdentures}

Overdentures are used as a treatment option in dentistry for more than 100 years. In 1856 Ledger constructed plates that covered the teeth and he referred the teeth to as fangs. Evans described the use of roots to preserve restorations in 1888, in 1896 Essig described the use of telescopic coping over intentional devitalization of roots. Dolder bars for over dentures were introduced in 1961. In 1969, Morrow, et al. narrated the simplified techniques of over dentures. In 1980, after the introduction of the phenomenon called osseintegration by Branemark et al, implant overdentures become an option [3]. 


\section{Prosthetic options for implant removable prosthesis}

Misch described five clinical prosthetic options in implantology. The first three are fixed prostheses. The other two types of final restorations are removable prostheses.

Removable prosthesis-type 4: RP-4 is a removable prosthesis completely supported by implants and/or teeth. The restoration is rigid when inserted; overdenture attachments connect the removable prosthesis to a low-profile tissue bar or superstructure that splints the implant abutments [4]. A minimum of five implants in the mandible and six to eight implants in the maxilla are required to fabricate completely implant-supported RP-4 prostheses [2]. Implant placement for an RP-4 prosthesis is different than for a fixed prosthesis. Denture teeth and acrylic bulk are required for the restoration. Superstructure and overdenture attachments must be added to the implants. This requires a more apical and lingual placement of the implant compared to that for an FP-1 or FP-2 prosthesis $[4,5]$. The position of an attachment on the superstructure may also affect the amount of spacing between the implants [5,6].

Removable prosthesis-type 5: RP-5 is a removable prosthesis with implant and soft tissue support. The amount of implant support is variable. The edentulous mandibular overdenture of this type may have, two anterior implants independent or splinted in the canine region to enhance retention. Three splinted implants in the anterior and premolar region to provide lateral stability. Four implants splinted with a cantilevered bar to reduce abrasions and to limit the soft tissue coverage needed for support. Naert, et al. described 10-year Randomized Clinical Trial on the Influence of Splinted and Unpainted Oral Implants Retaining Mandibular Overdentures [1]. The primary advantage of an RP-5 restoration is its reduced cost of treatment.

The bone however will continue to resorb in the soft tissueborne regions of the prosthesis. Relining and occlusal corrections every few years are common maintenance requirements. Bone resorption with an RP-5 may occur 2 to 3 times faster as compared with conventional complete dentures. This could be a factor to consider in the treatment of young patients despite the lesser cost [2]. No significant difference is found in stress reduction and peri implant health by using 2 implants or 4 implants. Sadowsky SJ, et al. described stress transfer of four implant overdenture cantilever designs [3]. Retention, stability, and occlusal equilibration improve only slightly by increasing the number of implants. Ohkubo C, et al. described ring attachments for transitional implants [5] Masticatory forces do not differ between the implant borne and the mucosa-implant-borne treatments [5].

\section{Overdenture movements}

The nature of motion of precision attachments used in implant overdentures may occur in zero to six directions: occlusal, gingival, facial, lingual, mesial, distal. However, the actual overdenture movement may be completely different from the one provided by independent attachments. Implant overdenture movement occurs in six directions or range of movements, rather than attachment directions. Sadowsky, et al. described load mechanisms in implant overdentures $[2,3]$. The prosthesis movement is evaluated when it is in the mouth. Prosthesis which is rigid in place and of a removable type, the prosthesis movement (PM) is identified as PM0 , regardless of the attachments used. For example, 0-rings have a range-of motion in six different directions. For example four 0-rings are placed along a complete arch bar, and the prosthesis rests on the superstructure bar, it may be a PM-0 restoration movement.

A hingelike prosthesis movement can occur in type 2 attachment for example, the Dolder bar without a space or Hader bar and clip. However, this system requires the attachment to be placed perpendicular to the axis of prosthesis rotation, so the prosthesis movement will also be in two planes PM-2. If the Hader bar is at an angle or parallel to the direction of desired rotation of the prosthesis, the prosthesis is rigid and has a PM-0. A crosssection of the Hader bar and clip system reveals that the system gains strength in comparison to a round bar design, by way of its design philosophy, also limits the amplitude of rotation of the clip and prosthesis around the fulcrum to around 20 degrees, thus transforming the prosthesis bar into a more rigid assembly.

Hence, bar and clip assembly is indicated for a PM-2 movement, when posterior ridges and the associated soft tissues are favourable to limit prosthesis rotation. A Hader bar-clip system is also an ideal low-profile attachment for RP-4 prosthesis, with PM-0. A Doldertype bar is desirable when greater amplitude of movement is needed to account for poorer ridge anatomy. Dolder bar, spacer, and clip assembly, permits apical movement and a hinge motion and categorised as Type 3 system. The bar and clip must also be perpendicular to the direction of prosthesis rotation to allow a PM-3 movement. Walton et al described 2 implant prosthetic designs [6].

A PM-4 restoration is rarely created with an overdenture system. The Type 4 overdenture attachment system allows a range of motion in a-mesial, distal, facial, and lingual direction, and the restoration may be removed. A magnet is the most common implant attachment system illustrating a four-direction movement. An advantage is that they exert practically no force on the implants. However, the implants usually must be independent from each other for this range of motion. If a superstructure connects the implants, the range of prosthesis motion decreases. magnets provide excellent retention but stability of the prosthesis needs refinement. Although great improvements in their size and retention characteristics have been achieved since their introduction into dentistry, magnets still often are characterised with the long-term problems of corrosion. $\mathrm{Chu}$, et al. described magnetic retained overdentures for Parkinson patient [7].

An 0-ring or ERA attachment may correspond to six directions of motion. However, the implants usually need to remain independent to permit a PM-6 range of movement. A superstructure bar will 
limit the prosthesis movement, depending on the design. A greater interarch space and moment of force are applied against the support system of an 0-ring because the vertical component of rotation for this attachment extends approximately $5 \mathrm{~mm}$ above the implant. Therefore Overdenture movement may be classified by evaluating the direction of movement of the implant supported prosthesis, and not by the overall range of motion for the individual attachments.

\section{Bone Density}

A Key Factor for Clinical Success. Besides its external architecture or volume, bone also has an internal architecture represented by its density. The strength or quality of bone supporting the endosteal implant is related directly to its density. Hence, bone density has a direct clinical influence on the success of implant treatment. anterior mandible bone density is greater than the anterior maxilla. The posterior mandible has lesser bone density than the anterior mandible. The poorest bone quality is often found in the posterior maxilla and is associated with greater failure rates. Although reports of implant survival has been found relative to this location, it is the bone density -quality encountered in these regions that influences implant success or failure. Overall, a higher failure rate is observed in poor quality bone than compared with higher quality bone, irrespective of the location.

Bone is an organ that changes in relation to a number of factors which could be physiologic (vitamins, hormones, minerals, supply of blood etc) or pathologic (trauma, tumour, genetic etc). In 1892 Wolff stated that: "Every change in the form and function of bone or of its function alone is followed by certain definite changes in the internal architecture, and equally definite alteration in its external conformation, in accordance with mathematical laws."

Cortical and trabecular bone throughout the body is modified constantly by modeling or remodeling. Modeling has independent sites of formation and resorption and results in the change of the shape or size of bone. It is mainly observed during early bone growth and during healing. Remodeling is a process of resorption and formation at the same site that replaces previously existing bone and primarily is responsible for the maintenance or homeostasis of bone (internal turnover). Both these adaptive phenomena are controlled primarily by the mechanical stress and strain environment within the host bone. Overall, the density of alveolar bone evolves as a result of mechanical deformation from microstrain. Bone is most dense around the teeth -cribriform plate and denser around the teeth at the crestal region compared with the regions around the apexes. A decrease in the mechanical strain environment over a period of time will lead to a reduction in the bone density, ex: around a tooth with no opposing occlusion or in regions of the maxilla or mandible after tooth loss.

Frost's Mechanostat theory, describes four zones of bone exist based on the mechanical adaptation to strain. Acute Disuse Window ( 0 to 50 microstrain), microstrain exerted is the least. Remodeling of bone is stimulated with greater resorption than formation taking place. The bone loses mineral density and disuse atrophy is seen to occur with a gradual net loss of bone. Adapted Window (50 to 1500 microstrain), This is an ideal physiologic loading zone with an equilibrium between the resorptive and formative phases of remodeling. This is the range of strain ideally desired around an endosteal implant. Mild Overload Zone (1500 to 3000 microstrain), micro-fractures and a stimulation of bone modelling occurs. However, the new bone formed is immature woven bone which is weaker. As a result, the bone strength and density eventually may decrease. Pathologic Overload zone (greater than 3000 microstrain), Remodeling with greater resorption of bone occurs due to the overload. If modeling does occur, then woven bone is formed because sustained repair is necessary. The crestal bone loss observed during early implant loading is due to pathologic overload.

\section{Lekholm and Zarb Bone Density Classification}

In 1985, Lekholm and Zarb listed four bone qualities found in the anterior regions of the jawbone.

1. homogenous compact bone,

2. Thick layer of compact bone surrounding a core of dense trabecular bone,

3. Thin layer of cortical bone surrounding dense trabecular bone of favourable strength,

4. Thin layer of cortical bone surrounding a core of lowdensity trabecular bone.

Irrespective of the different bone qualities, all bone was treated with the same implant design and standard surgical and prosthetic protocol. Different survival rates were accordingly reported. The clinical application of this classification system was questioned by Misch who in 1988, reported four bone density groups independent of the regions of the jaws based on macroscopic cortical and trabecular bone characteristics, implant design, surgical protocol, healing and progressive loading time spans have been described for each bone density type. Following this regimen, similar implant survival rates are observed for all bone densities.

\section{Misch bone density classification}

Cortical bone found on the outer surfaces of bone including the crest of an edentulous ridge may be either dense or porous. Similarly, trabecular bone found within the outer shell of cortical bone and occasionally on the crestal surface of an edentulous ridge may be either coarse or fine. A classification system based on these four macroscopic differences of bone has been proposed. D1 is Dense cortical bone, D2 is thick porous cortical bone on the crest and coarse trabecular bone, D3 is thin porous cortical bone on the crest and D4 is almost no crestal cortical bone and fine trabecular bone comprising all of the total volume of bone.

For treatment planning purposes, basic opinion regarding the bone density can be made based on the location. To err on the 
side of treatment planning for less dense bone is safer so that the prosthesis will be designed with slightly more, rather than less, support. Hence, Anterior mandible is usually treated as D2 bone; Posterior mandible as D3 bone, anterior maxilla as D3 bone, and posterior maxilla as $\mathrm{D} 4$ bone.

\section{Radiographic bone density}

Two dimensional radiographs like periapical or panoramic radiographs are not useful in identification of bone density, since cortical plates do not aid in revealing the trabecular bone density. In addition, the more subtle changes of D2 to D3 cannot be quantified by these radiographs. A more precise method is through the use of Computed Tomography or cone beam computed tomography. In general, the higher the CT number (Hounsfield unit), the denser the bone tissue. Computed Tomography Determination of Bone Density is described as D1->1250 HU, D2-850-1250 HU, D3-350-850 HU, D4-150-350 HU.

The initial bone density is responsible for the Mechanical immobilization of the implant during healing. Distribution and transmission of stresses from the prosthesis to the implant-bone interface after healing. Open marrow spaces or zones of unorganized fibrous tissue do not permit a controlled force dissipation. Also, the smaller the area of bone contacting the implant body, the greater the overall stress, when all other factors are equal. The bone density influences the amount of bone in contact with the implant surface, not only at first stage surgery but also at the second stage of uncovery and early prosthetic loading. bone contact is greater in cortical bone than in trabecular bone. Hence, a greater implant surface area is required to obtain a similar amount of bone-implant contact in softer bone compared with denser bone. Crestal bone loss and early implant failure after loading are mainly due to excess stress at the implant-bone interface. Thus, it is important to modify the treatment plans according to the bone density encountered in the potential implant site.

\section{Treatment planning}

In situations of decreased bone density, the biomechanical loads on the implants should be reduced definitely. Bone density is an important treatment plan modifier. Cantilever length may be shortened or eliminated. Narrower occlusal tables should be designed. Angle of load on the implant body should be more axial and offset loads minimized.RP-4 rather than FP restorations to be considered to reduce nocturnal parafunctional forces. RP-5 prostheses to be considered to permit the soft tissue to share the occlusal force. Night guards and acrylic occlusal surfaces distribute and dissipate the parafunctional forces on an implant system.

Increasing implant number is an excellent way to reduce stress by increasing the functional loading area.

Increasing the implant height in softer bone results in better initial fixation and healing. However, once initial healing is complete, the implant width is a more critical factor to reduce pathologic overload at the crestal region. D4 bone often requires wider implants compared with D1 or D2 bone. A different implant design is strongly suggested for each bone density because bone has a tenfold difference in strength and flexibility between D1 and D4 bone qualities. The deeper the thread, the more functional the surface area. A D4 implant body should have more and deeper threads than a D1 implant body. Coatings on an implant body increase the surface area. This provides for a gradual increase in occlusal loads, separated by a time interval to allow the bone to accommodate. Over time, progressive loading increases the density of bone at the implant interface and improves the overall support system mechanism. The softer the bone, the more important the progressive loading.

The mandible is well suited for implant retained overdentures, particularly when implants can be placed in the canine regions. Kimoto et al described Effect of Mandibular Ridge Height on Patients' Perceptions with Mandibular Conventional and ImplantAssisted overentures [8,9]. The greatest available height of bone is located in the anterior mandible, between the mental foramina or anterior loops of the mandibular canal when present. This region also usually presents the optimal density of bone for implant support.

The mental foramina are the posterior anatomical limitations in the anterior mandible and restrict fixture placement in this region. Two fixtures can be positioned bilaterally near these limits to provide cross-arch prosthesis stability. A third fixture can be placed in between the two fixtures. This is not always necessary, but can be done when the prognosis of one of the two fixtures is questionable. If one fails, there are still two fixtures remaining for use in the overdenture. If all three are successfully osseointegrated, then three fixtures can be used to support the overdenture. Anterior retention and stability for an overdenture offers several advantages. Therefore the treatment options presented are designed for anterior implant placement. The available bone of the anterior mandible is divided into five equal columns of bone serving as potential implant sites, labeled A, B, C, D, and E, starting from the patient's right. Regardless of the treatment option being executed, all five implant sites are mapped at the time of surgery.

The advantages of doing so are as follows: Patient always has the option of obtaining additional implant support in the future. For example, a patient may receive adequate support for an implant overdenture with four implants. However, if the patient desires a fixed prosthesis in the future, these four implants may fall short of the new requirements. If the implant surgeon did not plan an additional implant site at the initial surgery, but instead made the four implants an equal distance apart, the additional space may not be utilised without removal of one of the existing implants. Patient may desire a fixed prosthesis but is unable to afford the treatment all at once. Three implants in the A, C, and E positions and an overdenture may be provided now, and in the future two implants 
may be added in the $\mathrm{B}$ and $\mathrm{D}$ locations and a completely implant - supported overdenture or even a fixed restoration fabricated. If an implant complication occurs, the preselected option sites permit corrective procedures. This saves an additional surgery and eliminates the time required for bone healing before an implant could be reinserted. Therefore an organized pathway of treatment planning is provided for additional support if required in future or for the management of complications if it occurs.

\section{Advantages}

Retention and comfort, Cost effective. less number of implants compared to a fixed restoration. Convertibility to a conventional denture in case of implant failure. fixed restorations can be planned in future if the economic condition of the patient does not permits in the present. Increases the denture stability and chewing ability, so patient can take a broader array of nutrition. Stability of the implant overdenture keeps the muscle active and reduces muscle atrophy. Enhances psycologic confidence of the patient.

\section{Disadvantages}

Some overdentures can be bulky especially in a patient who has lost larger amounts of hard and soft supporting tissue because the denture base material is increased to compensate for the loss. Kreisler, et al. described bone resorption in maxillary implant overdentures [10]. Another disadvantage may involve the "removable" nature of this prosthesis. Patients who might have experienced a history of dissatisfaction with conventional denture use, may adamantly oppose another removable prosthesis although the prosthesis is secured to the implant. Chaffee et al described prosthetic complications in implant overdentures [11].

\section{Indications}

A younger segment of patients among the older population with conventional complete dentures. Elderly completely edentulous patients who request better stabilization of the existing conventional mandibular denture. Patients with acquired or congenital defects of maxilla or mandible [12]. Patients with post-surgical or congenital defects. These patients can be helped functionally and esthetically with this type of treatment. If either soft or hard tissue defects exist, esthetics can be improved by increasing or decreasing the amount of denture base material, The change in prosthetic design can enhance lip and facial support. Prosthesis stability and retention can be increased using attachments.

Patient who definitely wants osseointegrated implant treatment but lacks the dexterity necessary for hygienic maintenance of a fixed prosthesis. Implant hygiene maintenance can be less complicated by using fewer implants in overdenture prosthesis. The designs used for overdentures can be less cumbersome intra-orally than for a fixed prosthesis. If the patient later wants to convert to a fixed prosthesis, additional surgery may be done to place more implants in the remaining bone. In this case, the initial prosthesis can serve as an interim prosthesis through the subsequent phase of fabrication of a fixed prosthesis. Patients lacking good motor skills to adapt to wearing and chewing with conventional complete dentures. Completely edentulous patients who lose their teeth at an advanced age and are not capable of adapting to wearing conventional complete mandibular dentures. Loss of teeth at an advanced age presents a poor prognosis for learning new muscular patterns and can result in problems, for both the dentist and patient [13].

\section{Contraindications}

The patient is comfortable with existing complete dentures and presents no complaints. The residual ridge is inadequate for the standard placement of implants. History of Drug or Substance abuse. General health conditions which do not allow a minor surgical intervention. Ongoing therapy with corticosteroids or immunosuppressive drugs. uncontrolled metabolic disease.

\section{Discussion}

Since the prosthodontist cannot add additional abutments, when considering traditional prosthetic oral restorations, the restoration design is directly related to the existing oral conditions, which limits the treatment options. Implant dentistry can provide a number of abutments and as a result offer a range of treatment options to the partially and completely edentulous patients. Attard NJ, et al. described cost minimization analysis of implant overdentures [14]. Overdenture prosthetic treatment is an important alternative to a conventional mandibular or maxillary denture. A survey of elderly subjects showed that $66 \%$ were dissatisfied with their conventional complete dentures. The main reasons for dissatisfaction were discomfort, poor fit and retention, and especially soreness and pain under mandibular dentures, which caused many more problems than maxillary dentures [15].

Patients with mandibular overdentures retained by two implants had higher satisfaction scores than complete denture patients [2]. Naert, et al. described prosthetic aspects and patient satisfaction with Two-Implant Retained overdentures [16]. There are many advantages in choosing the overdenture osseointegration prosthesis. Fewer implants are required for prosthesis to function. Bouma described Psychosocial effects of implant retained overdentures [17]. Appointments are shorter, components costs are decreased, prostheses are less complicated Takanahi, et al. explained the fact that treatment is less expensive as compared to implant supported fixed restorations. Overdenture treatment should be the recommended treatment for patients with edentulous mandibles requesting implant-supported prostheses. This is of particular relevance to elderly patients in whom conventional complete denture adaptation is a predominant problem and whose limited physical and economic resources may prevent extensive treatment like implant fixed restorations [18].

Long term professional maintenance or treatment of complications is important in implant overdentures. Daily home care is easier and almost on par with conventional dentures home 
care. Kapur, et al. described a randomized clinical trial comparing the efficacy of mandibular implant-supported overdentures and conventional dentures in diabetic patient [19]. Facial esthetics can be enhanced with well supportive and retentive labial flanges and denture teeth compared with aging conventional denture flanges. The labial contours can replace lost bone width and height and support the labial soft tissues without hygienic compromise.

Prosthesis can be removed at night to manage parafunction if present in patient. Otherwise it can be worn at night also, unlike the conventional dentures. Payne, et al. described the prosthodontic maintenance requirements of mandibular mucosa-and implantsupported dentures [20]. Heydecke G, et al. described patient ratings in implant and conventional overdentures [13]. Mandibular implant overdentures were rated significantly easier to fabricate. Implant overdentures required fewer post-delivery adjustments to correct discomfort than conventional dentures. The clinician on average, spent about 15 minutes more adjusting conventional dentures [18]. The patient should not be encouraged to accept a fixed prosthesis if a removable prosthesis can predictably satisfy a patient's needs and desires [21].

\section{Conclusion}

Implant overdenture is very much a successful treatment option if the basics are understood in terms of bone science and patient selection. The limitations should be clearly explained to the patient at the diagnostic and discussion appointment so that any kind of misunderstanding of treatment and the prosthesis by the patient can be fully eliminated [22].

\section{Acknowledgement}

None.

\section{Conflict of Interest}

The authors declare no conflict of interest.

\section{References}

1. Naert I, Alsaadi G, van Steenberghe D, Quirynen M A (2004) 10-year Randomized Clinical Trial on the Influence of Splinted and Unsplinted Oral Implants Retaining Mandibular Overdentures: Peri-implant Outcome. Int J Oral Maxillofac Implants 19(5): 695-702.

2. Raghoebar GM, Meijer HJ, Slot W, Slater JJ, Vissink A (2014) A systematic review of implant-supported overdentures in the edentulous maxilla compared to the mandible: how many implants? Eur J Oral Implantol 7 Suppl 2: S191-201.

3. Sadowsky SJ (2001) Mandibular implant-retained overdentures: A literature review. J Prosthet Dent 86(5): 468-473.

4. Sadowsky SJ, Caputo AA (2004) Stress transfer of four implant overdenture cantilever designs. J Prosthet Dent 92(4): 328-336.

5. Ohkubo C, Sato J, Hosoi T, Kurtz KS (2004) O-Ring attachments for transitional implant retained overdentures. J Prosthet Dent 91(2): 195197.
6. Walton JN (2003) A Randomized Clinical Trial Comparing Two Mandibular Implant Overdenture Designs: 3 - Year Prosthetic Outcomes Using a Six-Field Protocol. Int J Prosthodont 16(3): 255-260.

7. Chu FCS, Deng FL, Siu ASC, Chow TW (2004) Soft-tissue supported, magnet-retained mandibular overdenture for an edentulous patient with Parkinson's disease: A clinical report. J Prosthet Dent 91(3): 219222 .

8. Kimoto K, Garrett NR (2005) Effect of Mandibular Ridge Height on Patients' Perceptions with Mandibular Conventional and ImplantAssisted Overdentures. Int J Oral Maxillofac Implants 20(5): 762-768.

9. Kimoto K, Garett NR (2003) Effect of mandibular ridge height on masticatory performance with mandibular conventional and implant assisted overdentures. Int J Oral Maxillofac Implants 18(4): 523-530.

10. Kreisler M, Behneke N, Behneke A, d'Hoedt B (2003) Residual Ridge Resorption in the Edentulous Maxilla in Patients with ImplantSupported Mandibular Overdentures: An 8-Year Retrospective Study. Int J Prosthodont 16(3): 295-300.

11. Chaffee NR, Felton DA, Cooper LF, Palmquist U, Smith R (2002) Prosthetic complications in an implant-retained mandibular overdenture population: Initial analysis of a prospective study. J Prosthet Dent 87(1): 40-44.

12. Mericske Stern R (1998) Treatment outcomes with implant-supported overdentures: Clinical considerations. J Prosthet Dent 79(1): 66-73.

13. Heydecke G, Klemetti E, Awad MA, Lund JP, Feine JS (2003) Relationship Between Prosthodontic Evaluation and Patient Ratings of Mandibular Conventional and Implant Prostheses. Int J Prosthodont 16(3): 307-312.

14. Attard NJ, Wei X, Laporte A, Zarb GA, Ungar WJ (2003) A Cost Minimization Analysis of Implant Treatment in Mandibular Edentulous Patients. Int J Prosthodont 16(3): 271-276.

15. Peitrokovski J, Harfin J, Mostavoy R, Levy F (1995) Oral findings in elderly nursing home residents in selected countries. Quality of and satisfacion with complete dentures. J Prosthet Dent 73(2): 132-135.

16. Naert I, Alsaadi G, Quirynen M (2004) Prosthetic Aspects and Patient Satisfaction with Two Implant Retained Mandibular Overdentures: A 10 Year Randomized Clinical Study. Int J Prosthodont 17(4): 401-410.

17. Bouma J, Boerrigter LM, Van Oort RP, Van Sonderen E, Boering G (1997) Psychosocial effects of implant-retained overdentures. Int J Oral Maxillofac Implants 12(4): 515-522.

18. Takanahi Y, Penrod JR, Chehade A (2002) Does a prosthodontist spend more time providing mandibular two-implant overdentures than conventional dentures? Int J Prosthodont 15(4): 397-403.

19. Kapur KK, Garett NR, Hamada MO (1998) A randomized clinical trial comparing the efficacy of mandibular implant-supported overdentures and conventional dentures in diabetic patients. Part I: Methodology and clinical outcomes. J Prosthet Dent 79(5): 555-569.

20. PayneAG, Solomons YF (2000) The prosthodontic maintenance requirements of mandibular mucosa-and implant- supported overdentures: a review of the literature. Int J Prosthodont 13(3): 238243.

21. Chaware SH, Thakkar ST (2020) A systematic review and meta-analysis of the attachments used in implant supported overdentures. J Indian Prosthodont Soc 20(3): 255-268.

22. Packer M, Nikitin V, Coward T, Davis DM, Fiske J (2009) The potential benefits of dental implants on the oral health quality of life of people with Parkinson's disease. Gerodontology 26(1): 11-18. 